\title{
Urine Cystatin C Determination in the Establishment of Reference Interval in the Diagnosis and Treatment of Renal Injury
}

\author{
Xiaoyu Jiang ${ }^{1 *}$, Lihua Qin ${ }^{2,3}$, Jing Wei ${ }^{2,3}$, Guosheng Su${ }^{2,3}$, Xiaoye Su${ }^{4,5}$, Aihua $\mathrm{Lu}^{2,3}$, \\ Jiansheng Huang2,3, Feng Lu' ${ }^{2,3}$, Taixin $\mathbf{L u}^{2,3}$, Pinchun Huang ${ }^{2,3}$, Buqing Su6 \\ ${ }^{1}$ Department of Laboratory Medicine, Third People's Hospital of Nanning, Nanning, China; ${ }^{2}$ Guangxi-ASEAN \\ Economic and Technological Development Zone People's Hospital, Nanning, China; ${ }^{3}$ The Department of Nephrology \\ \& Laboratory Medicine of Tenth People's Hospital of Nanning, Nanning, China; ${ }^{4}$ Department of Nursing, Graduate \\ School of Everlasting, University of the Philippines, Fuzhou, China; ${ }^{5}$ Department of Critical Care Medicine, Fuzhou \\ Second People's Hospital, Fuzhou, China; ${ }^{6}$ Department of Nursing, Liaoning Vocational College of Medicine, \\ Shenyang, China
}

Correspondence to: Jing Wei, 247535274@qq.com; Guosheng Su, 563449581@qq.com

Keywords: Urine, Cystatin C, Kidney Injury, Concentration Interval, Standardization

Received: October 29, $2021 \quad$ Accepted: January 4, $2022 \quad$ Published: January 7, 2022

Copyright $\odot 2022$ by author(s) and Scientific Research Publishing Inc.

This work is licensed under the Creative Commons Attribution International License (CC BY 4.0).

http://creativecommons.org/licenses/by/4.0/

\section{(c) (i) Open Access}

\section{ABSTRACT}

Objective: To establish the diagnostic concentration range of urine cystatin $\mathrm{C}$ and the control level of urine cystatin $C$ for patients with renal injury, and to help promote the establishment of standardization of urine cystatin C detection. Methods: 150 urine specimens and blood specimens from kidney injury patients and healthy people were collected, and stored in the refrigerator at $-80^{\circ} \mathrm{C}$ for later use. After the specimens were collected, they were uniformly tested. Comparing the difference of blood and urine cystatin $\mathrm{C}$ between the kidney injury group and the healthy control group, the application value of urine cystatin $\mathrm{C}$ in the diagnosis and treatment of kidney injury was put forward. Results: The concentrations of cystatin C in urine and blood of renal injury group were $1.04 \pm 2.14 \mathrm{mg} / \mathrm{L}$ and $1.94 \pm 2.36 \mathrm{mg} / \mathrm{L}$ respectively; the concentrations of cystatin $\mathrm{C}$ in urine and blood of healthy control group were 0.11 $\pm 0.05 \mathrm{mg} / \mathrm{L}$ and $0.83 \pm 0.20 \mathrm{mg} / \mathrm{L}$ respectively. The urine and blood cystatin $\mathrm{C}$ of the kidney injury group were significantly higher than those of the healthy control group. Compared the results of the two groups, the $t$ values were 5.3210 and 5.7399 , respectively. The $P$ value is 0.0000; the difference is statistically significant; in comparison of urine and blood cystatin C in the kidney injury group, the value is that $t=3.4600, P=0.0003$; in comparison of urine and blood cystatin $C$ in the healthy control group, the value is that $t=42.7744, P=0.0000$. By

${ }^{\star}$ Tied to the first author. 
investigating the urine cystatin $\mathrm{C}$ concentration of patients with kidney injury, whether it is kidney injury or healthy controls, urine cystatin $C$ is significantly lower than blood cystatin C. Conclusion: According to the results of this study, the concentration of urinary cystatin $\mathrm{C}$ in the healthy control group is $0.11 \pm 0.05 \mathrm{mg} / \mathrm{L}$. The reference interval of urinary cystatin $C$ proposed in this study is $0.06-0.16 \mathrm{mg} / \mathrm{L}$, which can be established by comprehensive multi center research. Through a multi-center study of the baseline level of urinary cystatin $\mathrm{C}$ in patients with renal injury, the diagnostic concentration interval of urinary cystatin $\mathrm{C}$ and the control level of urine cystatin $\mathrm{C}$ for renal injury patients were established.

\section{INTRODUCTION}

Cystatin $\mathrm{C}$ is a small molecular weight cystine protease inhibitor, which is expressed by all nucleated cells in the body with a constant production rate [1]. Under normal physiological conditions, Cystatin C can be freely filtered from the glomerulus with plasma, and can be completely reabsorbed by renal tubular epithelial cells and degraded in the cell without returning to the blood; however, tubular epithelial cells do not secrete cystatin $\mathrm{c}$ to the lumen. Therefore, serum cystatin $\mathrm{C}$ is used as a functional indicator of glomerular filtration rate [2-6]. However, when renal tubular function is impaired, cystatin $C$ is impaired in renal tubular reabsorption, and the concentration of cystatin $\mathrm{C}$ in the urine increases. At this time, cystatin $\mathrm{C}$ in urine is used as an important indicator for evaluating renal tubular function. However, at present, the detection of renal function is mostly based on the detection of serum cystatin C. The recent findings of primary glomerular diseases or renal vascular diseases and other diseases are also accompanied by different degrees of tubular interstitial damage, and the severity of damage is directly related to the speed of development and prognosis of kidney disease. At this time, detecting the concentration of cystatin $\mathrm{C}$ in the urine is a very sensitive indicator reflecting the damage of the renal tubules [7-9]. However, there are still controversies about the standardization and uniform reference interval for the detection of urinary cystatin $\mathrm{C}$ in clinical practice. Therefore, this research group investigated the urine cystatin $\mathrm{C}$ concentration of patients with kidney injury, and calculated the baseline level of urine cystatin $\mathrm{C}$ in patients with kidney injury; established the diagnostic concentration interval of urine cystatin $\mathrm{C}$ and the control level of urine cystatin $\mathrm{C}$ for patients with kidney injury, to help promote the establishment of standardization of urine cystatin C detection.

\section{MATERIALS AND METHODS}

\subsection{Research Subjects}

150 urine and blood specimens of patients who were hospitalized in this hospital from January to September 2021 and confirmed kidney injury were selected as the research subjects, that is, the kidney injury group, and other basic diseases were excluded; In addition, 150 urine and blood specimens of healthy people who underwent physical examinations in the physical examination center of this hospital during the same period were selected as the control group, which is the healthy control group. The basic data of the two groups, such as gender, age, occupation, etc., are basically the same. Urine samples were collected according to the sample collection specification and stored in $-20^{\circ} \mathrm{C}$ refrigerator; Blood samples were collected by taking $2 \mathrm{ml}$ of fasting venous whole blood, centrifuging at $4000 \mathrm{rpm}$ for 10 minutes, and the serum was stored in the refrigerator at $-20^{\circ} \mathrm{C}$. After all samples were collected, they were tested uniformly. The research will be carried out after the approval of the medical ethics committee of the hospital.

\subsection{Research Methods}

Research object grouping: 1) Healthy control group: 150 cases, aged 17 to 70 years old, excluded hypertension, diabetes, hyperlipidemia, cardiovascular, kidney and other diseases that affect renal function, and blood and urine routines are normal Healthy people. 2) Collect at least 150 patients with kidney 
disease as judged by "Diagnosis of Kidney". 3) Detect the urine and blood cystatin C levels of the above two groups, and analyze the urine cystatin $\mathrm{C}$ concentration range of patients with different degrees of nephropathy. Compare with the detection method of serum cystatin $C$ to determine the control level of urinary cystatin C.

\subsection{Implementation Plan}

Collect urine specimens of all research subjects, and use the kit of Beijing Jiuqiang Biotechnology Co., Ltd. to detect the level of cystatin C. This topic mainly uses the automatic biochemical analyzer Hitachi 7180 to test patient samples and issue test results.

\subsection{Reference Interval}

The reference interval of serum cystatin $\mathrm{C}$ is $0.6-2.5 \mathrm{mg} / \mathrm{l}$.

\subsection{Statistical Analysis}

The SPSS22.0 statistical software was used for analysis. The comparison of the number of cases between the two groups was performed by chi-square test; the comparison of cystatin $\mathrm{C}$ values was performed by $\mathrm{t}$ test, and $\mathrm{P}<0.05$ was considered statistically significant.

\section{RESULTS}

After testing the blood and urine cystatin $\mathrm{C}$ of 300 study subjects, it was found that the urine and blood cystatin $\mathrm{C}$ of the kidney injury group were significantly higher than those of the healthy control group. Compared the results of the two groups, the $t$ values were 5.3210 and 5.7399 , respectively. The $\mathrm{P}$ value is 0.0000 , the difference is statistically significant; the comparison of urine and blood cystatin $\mathrm{C}$ in the kidney injury group, $\mathrm{t}=3.4600, \mathrm{P}=0.0003$; the comparison of urine and blood cystatin $\mathrm{C}$ in the healthy control group, $\mathrm{t}=42.7744, \mathrm{P}=0.0000$.

The specific results are shown in Table 1 below.

\section{DISCUSSION}

Cystatin $\mathrm{C}$ is an alkaline, non-glycosylated protein, which can be expressed constantly and continuously in all cell nuclei and does not fluctuate over time. Cystatin $\mathrm{C}$ can freely pass through the glomerular filter membrane in the physiological environment, and is almost completely reabsorbed and degraded in the renal tubules, without recirculation, and the renal tubules will not secrete Cystatin C. Under normal physiological conditions, the concentration of cystatin C in urine is very low, only $5 \%$ to $10 \%$ in blood. However, when the renal tubules are damaged and dysfunction occurs, the concentration of cystatin $\mathrm{C}$ in the urine increases [10].

The clinical evaluation of renal function indicators is generally based on the glomerular filtration rate as the standard. Markers of endogenous glomerular filtration rate are serum creatinine, urea, $\beta 2$-microglobulin, $\beta$-trace protein and cystatin C. Among them, Cystatin C is more reliable and accurate than other indicators.

Table 1. The application of urine cystatin $\mathrm{C}$ in renal impairment.

\begin{tabular}{cccccc}
\hline Group & Number of cases & Urine CYS-C & Blood CYS-C & t value & P value \\
\hline Kidney injury group & 150 & $1.04 \pm 2.14$ & $1.94 \pm 2.36$ & 3.4600 & 0.0003 \\
Healthy control group & 150 & $0.11 \pm 0.05$ & $0.83 \pm 0.20$ & 42.7744 & 0.0000 \\
t value & - & 5.3210 & 5.7399 & - & - \\
P value & - & 0.0000 & 0.0000 & - & - \\
\hline
\end{tabular}


Most of the current cystatin $\mathrm{C}$ detection methods and samples are mainly serum cystatin $\mathrm{C}$, but cystatin $\mathrm{C}$ in urine samples are less studied, and the standardization and stability of urine cystatin $\mathrm{C}$ detection and the unified reference interval are clinically controversial $[11,12]$. Therefore, this research group investigated the urine cystatin $\mathrm{C}$ concentration of patients with kidney injury, and calculated the baseline level of urine cystatin $\mathrm{C}$ in patients with kidney injury, and established the diagnostic concentration interval of urinary cystatin $\mathrm{C}$ and the control level of urine cystatin $\mathrm{C}$ for patients with kidney injury, and help promote the establishment of standardization and stability of urine cystatin $\mathrm{C}$ detection.

The results of this study showed that the blood and urine cystatin $\mathrm{C}$ of the kidney injury group was significantly higher than that of the healthy control group. The difference between the two groups was statistically significant, $\mathrm{P}<0.001$; the blood cystatin $\mathrm{C}$ of the two groups was significantly higher than the urine Cystatin C; blood cystatin $\mathrm{C}$ and urine cystatin $\mathrm{C}$ were compared between the two groups, and the differences were statistically significant, $\mathrm{P}<0.01$. The reference interval of urinary cystatin $\mathrm{C}$ is lower than that of blood, and can be established uniformly based on multi-center studies.

\section{CONCLUSION}

By investigating the urine cystatin $\mathrm{C}$ concentration of patients with kidney injury, whether it is kidney injury or healthy controls, urine cystatin $\mathrm{C}$ is significantly lower than blood cystatin $\mathrm{C}$. Through multicenter study of the baseline levels of urinary cystatin $\mathrm{C}$ in the population of kidney injury patients, it establishes the diagnostic concentration interval of urinary cystatin $\mathrm{C}$ and the control levels of detecting urine cystatin $\mathrm{C}$ in kidney injury patients, to help promote the detection standardization and stability establishment of urinary cystatin C.

\section{LIMITATIONS OF THE STUDY}

All the research subjects in this study are from the inpatients in this hospital and the healthy population of the physical examination center. There are certain regional differences and it cannot fully represent the situation in other regions, so it has certain limitations.

\section{ACKNOWLEDGEMENTS}

This project has been approved, and has received strong support from the functional departments of the hospital and the help of colleagues. I would like to express my heartfelt thanks for their support and help! I wish them good health, smooth work and all the best!

\section{FUNDING PROJECT}

The clinical application value of urine sample detection CYS-C as a sub-project of the national key research and development plan "National Quality Basic Common Technology Research and Application" key special geriatric-related biochemical diagnostic reagent quantity traceability and quality evaluation research (NO.: 2019YFF0216502).

\section{CONFLICTS OF INTEREST}

The authors declare no conflicts of interest regarding the publication of this paper.

\section{REFERENCES}

1. Xu, Q. (2020) The Predictive Value of Cystatin C in the Early Diagnosis of Diabetic Nephropathy. Jilin University, Jilin.

2. Xie, S.Q., Gong, M., Liu, W., et al. (2019) The Clinical Significance of Serum Cystatin C and Urine Renal Injury Molecule-1 in Patients with Early Renal Injury in Hypertensive Disorders of Pregnancy. Experimental and La- 
boratory Medicine, 37, 356-357+371.

3. Chen, S.P. (2021) The Diagnostic Value of Serum Cystatin C and Urine Renal Damage Molecule-1 in the Diagnosis of Early Renal Injury in Patients with Hypertension in Pregnancy. China Maternal and Child Health Care, 36, 555-557.

4. Fan, L.J., Lu, T.J. and Cui, Y.J. (2019) Urine Complement Factor D Combined with Serum Cystatin C to Predict the Value of Severe Preeclampsia Renal Injury. China Journal of Modern Medicine, 29, 113-116.

5. Wang, G.Q., Yang, L.H., Cheng, X., et al. (2018) The Diagnostic Value of Urine Microprotein, Cystatin C and Urine Routine Tests for Early Renal Function Damage in Patients with Hyperbilirubinemia. Journal of Modern Laboratory Medicine, 33, 70-73.

6. Chen, Y.P., Huang, L. and Wu, J.Q. (2018) The Evaluation Significance of Serum Cystatin C and Urine Kidney Injury Molecule 1 Levels on the Degree of Renal Tubulointerstitial Injury in Hypertension. Clinical Medical Engineering, 25, 1077-1078.

7. Wang, B. (2018) To Explore the Clinical Value of Combined Detection of Urinary Microalbumin/Creatinine and Cystatin C in the Early Diagnosis of Diabetic Nephropathy. Medical Treatment, No. 1, 73.

8. Huang, L.L., Miao, H.J., Lu, Y., et al. (2017) Changes in Serum Cystatin C and Urine Renal Injury Molecule 1 Levels in Patients with Sepsis and Their Diagnostic Value for Complicated Acute Kidney Injury. Shandong Medicine, 57, 70-72.

9. Wang, H., Lu, Y., Gu, Y.J., et al. (2017) Establishment and Evaluation of the Detection Limit and Quantification Limit of Urine Cystatin C with Conventional Cystatin C Reagent Based on EP17-A Document. Shanxi Medical Journal, 46, 1224-1226.

10. Zhou, W. and Zhou, L.L. (2021) The Clinical Value of Urine Cystatin C Combined with Serum $\beta \_2$ Microglobulin Level in the Diagnosis of Patients with Lupus Nephritis. Labeled Immunoassay and Clinic, 28, 1185-1187+1221.

11. Zeng, Y., Zhang, L., Shen, H., et al. (2019) The Clinical Value of Serum Homocysteine, Cystatin C, Urine Albumin, and Urine $\beta \_2$ Microglobulin Levels on Diabetic Nephropathy. Journal of Practical Clinical Medicine, 23, 109-112.

12. Li, Z.Q. and Li, H.B. (2019) Analysis of the Diagnostic Value of Serum Cystatin C Combined with Urine a1-MG and mALB in the Diagnosis of Early Renal Damage in Chronic Nephritis. Harbin Medicine, 39, 304-306. 